\title{
Cough and Hemoptysis in a Middle-Aged Woman
}

\author{
Haiyan Ge ${ }^{a, b}$ David Garfield ${ }^{d}$ Suqin Ben ${ }^{b}$ Jianguo Zhang ${ }^{c}$ Ying Liu ${ }^{c}$ \\ Jun $\mathrm{Gu}^{\mathrm{b}}$ Songshi $\mathrm{Ni}^{\mathrm{b}}$ Chunxue $\mathrm{Bai}^{\mathrm{a}}$ \\ ${ }^{a}$ Department of Pulmonary Medicine, Zhongshan Hospital, Fudan University, Shanghai, Departments of \\ ${ }^{b}$ Respiratory Medicine and ${ }^{C}$ Pathology, Affiliated Hospital of Nantong University, Nantong, and d Promed Cancer \\ Centers, Shanghai, China
}

\section{Case Presentation}

A 44-year-old woman with a 3-month history of cough and more recent hemoptysis was admitted to the respiratory ward at the affiliated hospital of Nantong University. Her cough had not responded to empiric antibiotic therapy. One week prior to admission, she noted worsening of the cough and development of hemoptysis. For the prior half year, she had frequent mild aches in the lower limbs and minor swelling of knee joints. She denied fevers, chills, chest pain, night sweats, weight loss, or loss of appetite. The patient was a nonsmoker and denied use of alcohol, illicit drugs, or exposure to toxins. Her family history was negative. Prominent physical findings included decreased respiratory sounds and rales in the right lung, and digital clubbing of fingers (fig. 1).

A frontal chest radiograph showed a large, confluent, homogenous mass in the right lower lung. It had sharply demarcated superior and inferior margins with an angle towards the pleura. A contrast-enhanced CT scan dem-

H. Ge and D. Garfield contributed equally to this work. onstrated that the orifice and lumen of the right middle lobe bronchus were significantly obstructed by an irregular mass (fig. 2). There were no mediastinal or pleural abnormalities.

Bronchoscopy revealed a large gray mass subtotally obstructing the right middle lobe bronchus (fig. 3). This was biopsied without incident. Histopathological examination showed sheets of spindle-shaped, pleomorphic cells having vesicular nuclei, prominent nucleoli, and brown cytoplasmic pigment (fig. 4).

Radiography of the ulna, radius, tibia, and fibula revealed periosteal membrane thickening and periosteal new bone formation (fig. 5). A Tc-99m MDP bone scan showed increased uptake in the periosteal regions of long bones and osteoarthritis of extremities (fig. 6).

What Is Your Diagnosis?

Corresponding authors are:

Chunxue Bai (adress see below) and Songshi Ni, MD

Affiliated Hospital of Nantong University

Nantong 226001 (China)

Tel. +865138116 1606, E-Mail jsntnss@163.com

Chunxue Bai, MD, PhD, FCCP

Department of Pulmonary Medicine

Zhongshan Hospital, Fudan University

Shanghai 200032 (China)

Tel. +86 216404 1990, E-Mail bai.chunxue@zs-hospital.sh.cn 
Fig. 1. Digital clubbing of fingers - expansion with broadened and thickened fingertips.
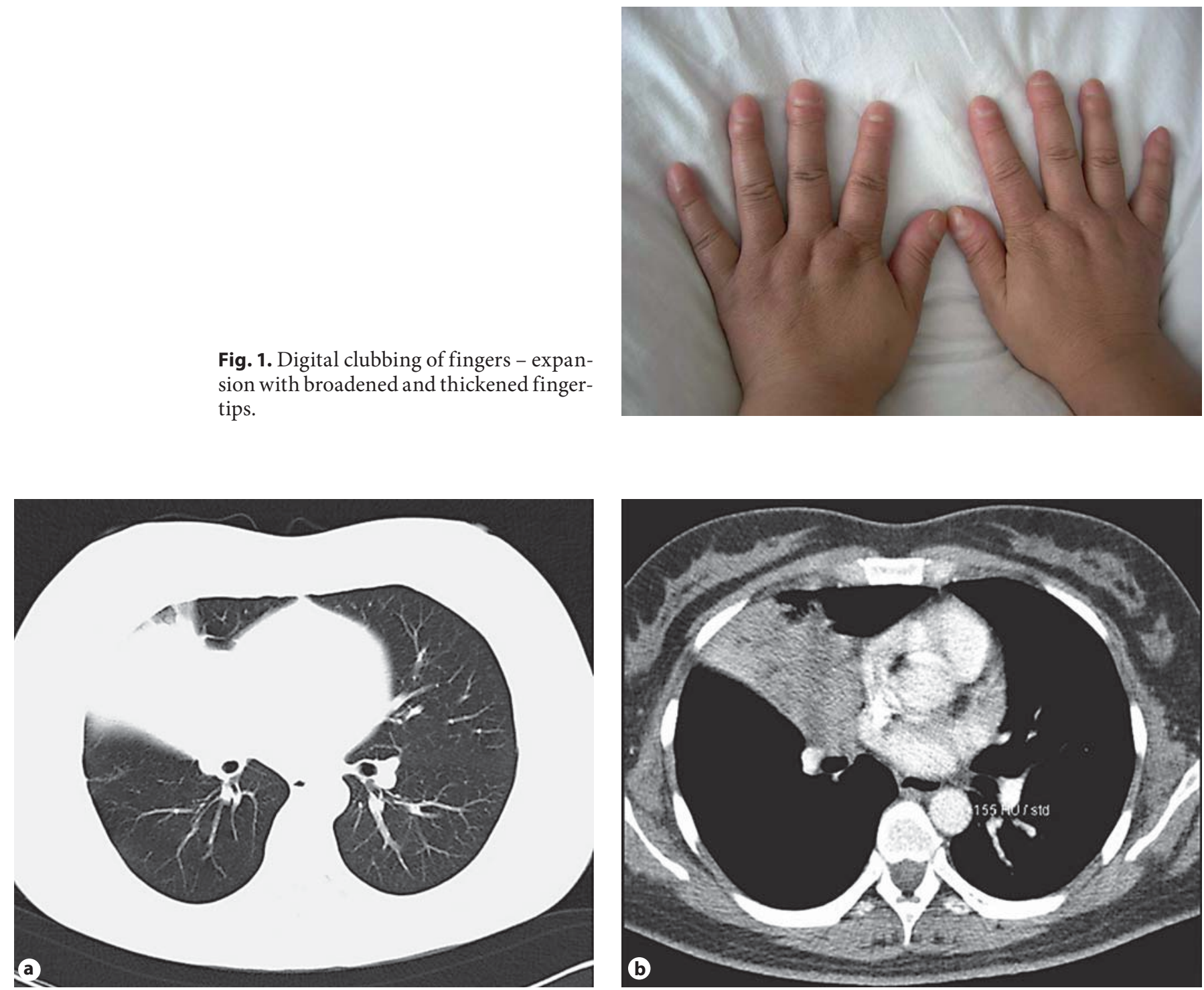

Fig. 2. a, b Initial chest CT scan showing the orifice of the right middle lobe bronchial lumen to be narrowed and partially obstructed by an irregular mass.

Fig. 3. Bronchoscopy showing a large gray mass almost totally obstructing the right middle lobe bronchus.

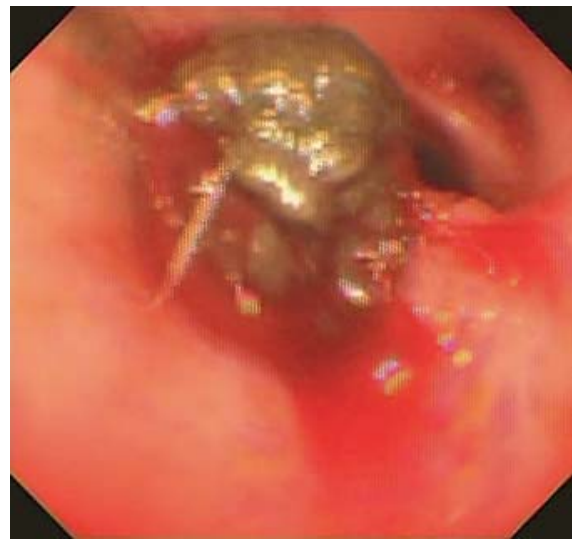




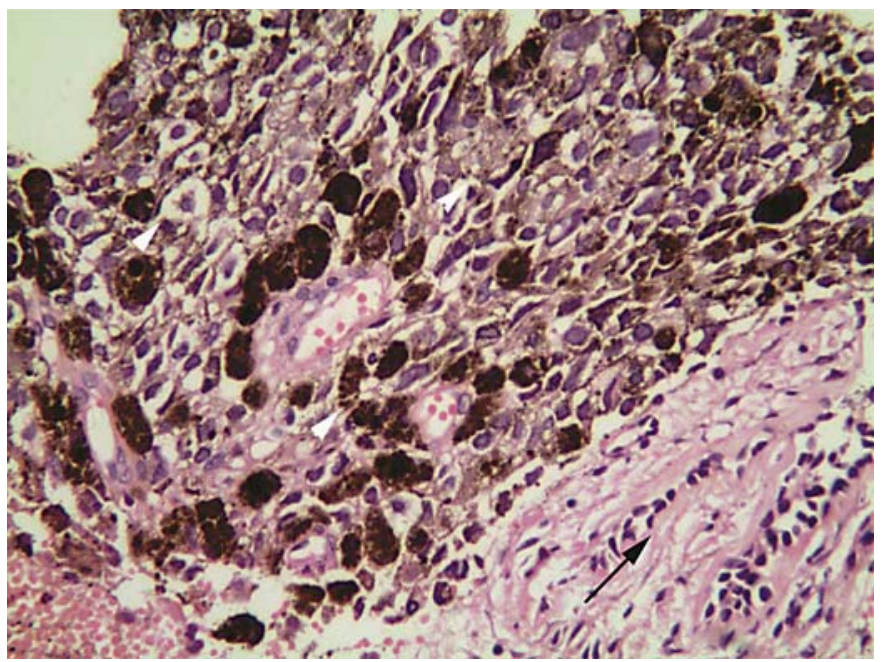

Fig. 4. Transbronchial biopsy specimen. Photomicrograph showing sheets of spindle-shaped, pleomorphic cells having vesicular nuclei, prominent nucleoli, brown cytoplasmic pigment (arrowhead), and normal lung tissue around (arrow). Hematoxylin-eo$\sin \times 200$.

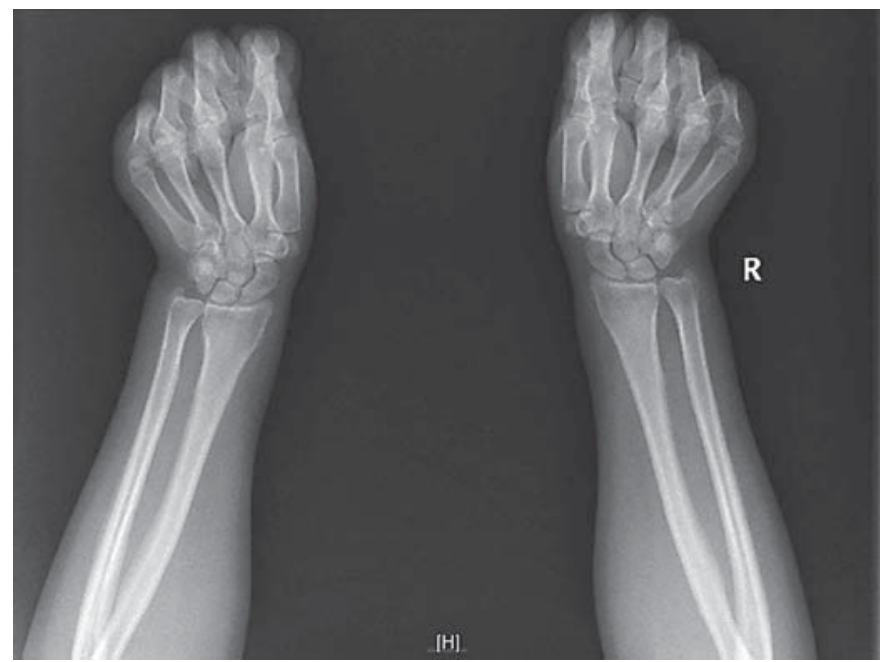

Fig. 5. Bone radiography revealing periosteal membrane thickening and periosteal new bone formation of the ulna and radius.
Fig. 6. Anterior (a) and posterior (b) views of bone scans showing symmetric diffuse cortical uptake around the diaphysis in both tibias and femurs.

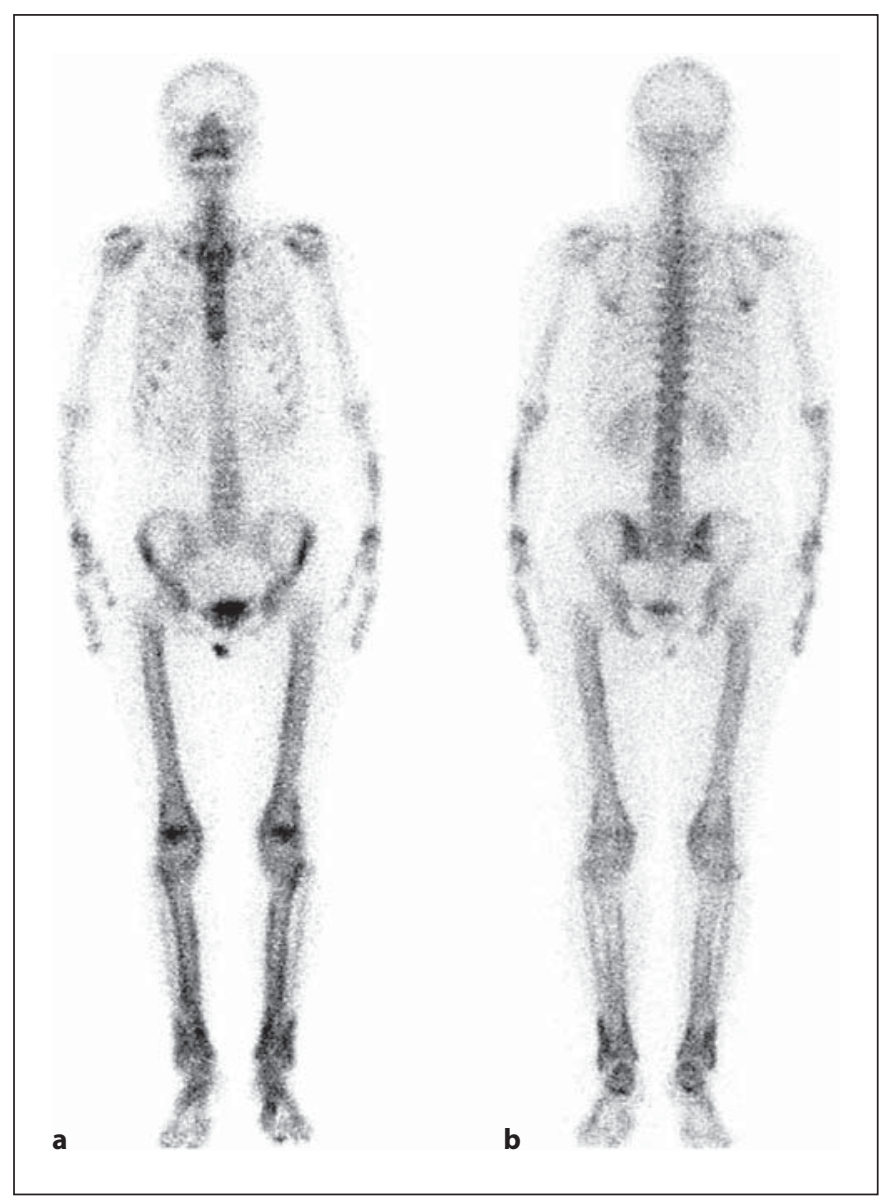

Respiration 2012;83:357-362 

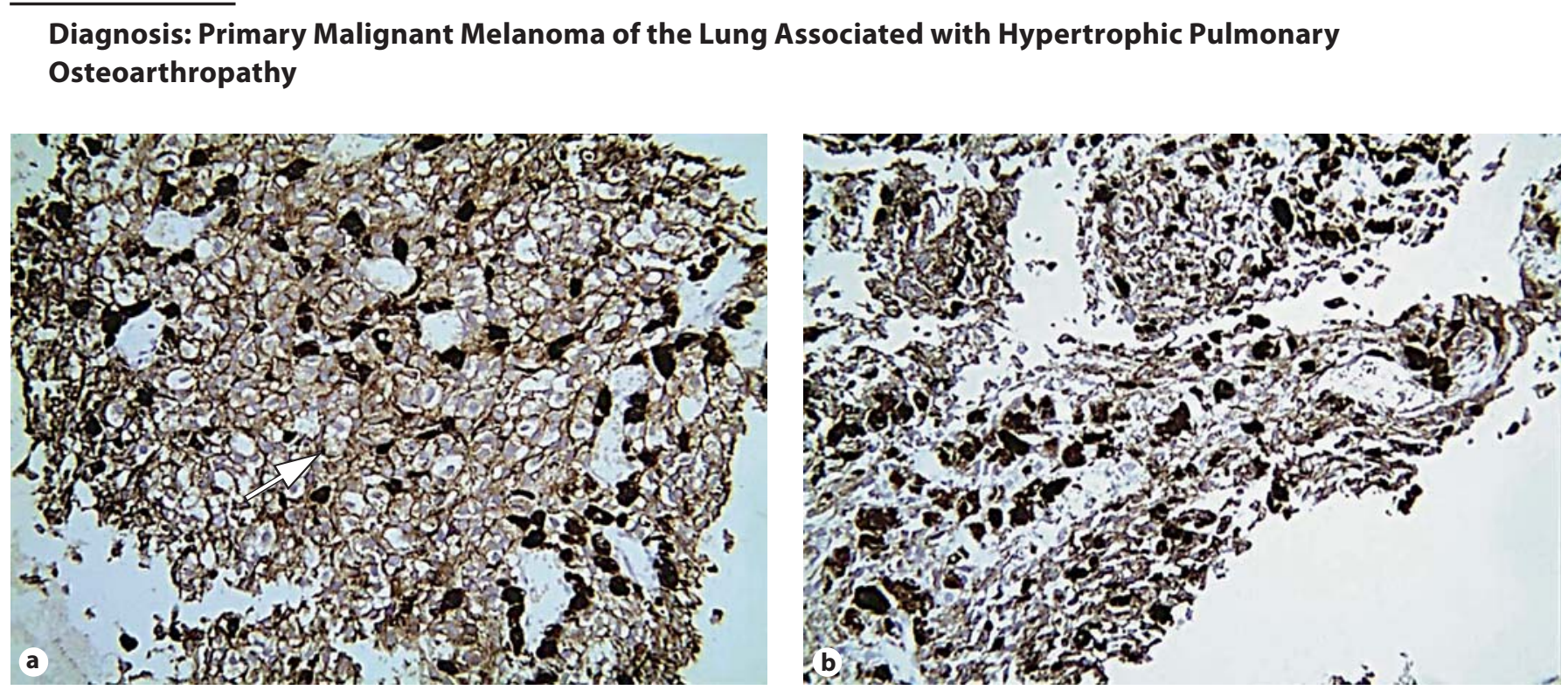

Fig. 7. a Transbronchial biopsy specimen. Photomicrograph showing immunohistochemical staining of melanoma cells with antibody HMB45 which is mainly expressed in cell plasma (arrow; Avidin-Biotin Complex $\times 200$ ). b Photomicrograph showing immunohistochemical staining of melanoma cells with antibody S100 (Avidin-Biotin Complex ×200).

\section{Clinical Course}

As coughing and anti-inflammation therapy were ineffective, a diagnostic flexible video-bronchoscopy was performed in order to obtain biopsy specimens. Immunohistochemistry showed that the malignant cells were positive for S100 and HMB45 (fig. 7), whereas cytokeratin, chromogranin, and CD68 were negative. Subsequently, a more detailed history and thorough physical examination was carried out after the histopathological diagnosis. The patient did not have previous cutaneous or mucosal lesions excised. Examinations of the retina, ears, nose, nasopharynx, anus, and esophageal mucosa also failed to detect any abnormalities. All blood tests were within normal limits, including creatinine kinase, aldolase, Sjögren's syndrome antigens (SSA/SSB), antinuclear antibody, and rheumatoid factor. With all the above evidence, the patient was diagnosed as having primary malignant melanoma of the lung (PMML) associated with hypertrophic pulmonary osteoarthropathy (HPO).

After the definitive diagnosis was made, the patient was advised to have surgery. However, she and her family chose, instead, to have $\gamma$-knife treatment at another hospital. After $\gamma$-knife treatment, the tumor size was reduced by about $30 \%$. She also received chemotherapy with dacarbazine, high-dose interleukin-2, and interferon. The patient had monthly follow-ups, and after 3 months of chemotherapy she had relief of cough and no hemoptysis. Chest CT showed stable disease. However, she developed headaches 1 year after the diagnosis and was found to have brain and lung metastases.

\section{Discussion}

This case presents a patient who has PMML associated with HPO. Malignant melanoma is one of the most lethal cancers and has been documented to originate in various anatomic locations, although most are cutaneous. Extracutaneous malignant melanomas are rare entities that can originate from squamous mucous membranes, eyes, esophagus, leptomeninges, oral cavity, larynx, etc. PMML is a quite rare neoplasm, accounting for $0.01 \%$ of all lung tumors, with less than 35 cases described in the English and non-English literature $[1,2]$. The patients often present with cough, hemoptysis, obstructive pneumonia, lobar collapse, or atelectasis. The clinical presentation and radiological appearance is similar to lung cancer. Bronchoscopic biopsy usually makes the diagnosis [3], as in the present case.

The diagnosis of PMML is established based on rigid clinical, radiological, and pathological findings. These 
include [4]: (1) junctional changes described as 'dropping off' or 'nesting' of melanoma cells just beneath the bronchial epithelium; (2) invasion of the bronchial epithelium by melanoma cells; (3) malignant melanoma associated with these epithelial changes; (4) a solitary lung tumor with morphology compatible with a primary tumor; (5) no history of cutaneous, mucous membrane, or ocular melanomas; and (6) absence of any other detectable tumor at the time of diagnosis. Taking these criteria into account, our case carried the specification for primary tumor morphology.

The main radiographic feature of primary lung cancer is a pulmonary nodule/mass, as with PMML [5]. PMML can be differentiated from metastatic malignant melanoma of the lung in that the pulmonary nodule/mass is solitary, central, and often obstructs a bronchus. Histopathologically, PMML often shows epithelial junctional changes, with 'dropping off' or 'nesting' of melanoma cells beneath the bronchial epithelium, invasion of bronchial epithelium by melanoma cells in ulcerated areas, and with the mass often appearing as a solitary tumor [6]. The diagnosis also depends on histopathologic appearance and immunoreactivity with $\mathrm{S} 100$ protein and melanocytic markers, including HMB45, melan-A, and PNL2. In this case, histopathological examination showed sheets of spindle-to-pleomorphic cells with vesicular nuclei, prominent nucleoli and brown cytoplasmic pigment. Immunohistochemistry showed malignant cells which were positive for S100 and HMB45, whereas cytokeratin, chromogranin, and CD68 were negative. Based on the patient's choice, we did not additionally obtain a surgical specimen, which may have been more convincing. However, the above results still confirmed, we believe, the diagnosis of primary pulmonary malignant melanoma.

Theories of how primary malignant melanoma arises in the lung are controversial since melanocytes are not present in the epithelium of the normal bronchus. There are several theories which attempt to provide an explanation. One theory suggests that the tumor arises from melanocytes that have migrated along with the primordial tubular respiratory tract during embryogenesis [7]. Epithelial cell damage and transformation play important roles in lung cancer development [8], so another theory is that respiratory epithelial cells undergo metaplastic transformation into melanocytes, termed 'melanogenic metaplasia' [9]. It has also been proposed that other cell types, such as neuroendocrine precursor cells, have the potential to undergo melanocytic transformation [10].

Cough and Hemoptysis in a Middle-Aged Woman
HPO constitutes a clinical syndrome characterized by (1) clubbing of fingers and toes, (2) arthralgias, and (3) periostosis of distal long bones, especially the anterior tibias. It may be accompanied by thickening of subcutaneous soft tissues in distal parts of extremities and sometimes of the face and scalp, as well as by neurovascular changes of hands and feet. In most instances, HPO occurs secondarily to conditions characterized by an arteriovenous shunt, such as lung carcinoma, mesothelioma, pulmonary tuberculosis, or congenital cyanotic heart disease; it has also been associated with hepatic and colorectal carcinoma, inflammatory bowel disease, cirrhosis, pulmonary fibrosis, and pleural empyema [11]. A recent retrospective study [12] indicated that $4.5 \%$ of primary lung cancer patients showed findings suggestive of HPO, but with such patients rarely showing the full triad of the above signs and symptoms. Cases of malignant melanoma metastatic to the lung associated with HPO [13] have been reported, but PMML associated with HPO has never been reported to our knowledge. Our patient fulfilled HPO criteria with the HPO due to primary invasive malignant melanoma of the lung.

Periostitis is a hallmark of HPO, with bone radiography revealing periosteal membrane and periosteal new bone formation. Tc-99m MDP bone scan is a useful, readily available imaging procedure that may facilitate the diagnosis of HPO. The scan shows bracelet-like or diffusely increased uptake throughout most tubular bones. Interestingly, in half of the lung cancer patients, the bone scan findings of HPO improved with treatment of the lung cancer [14]. In our case, it is not known if the HPOrelated imaging studies showed improvement.

The pathogenesis of HPO remains not fully understood. Immunologic, hormonal, neural, and neurohumoral factors have been implicated. A potential mechanism for its development is increased circulating growth factor levels, especially vascular endothelial growth factor (VEGF). VEGF, a powerful endothelial cell-stimulating factor, very possibly plays a central role in the pathogenesis of HPO for several reasons [15]. One study [16] showed that unfragmented megakaryocytes can stimulate production of platelet-derived growth factor and VEGF by endothelial cells, resulting in neoangiogenesis and endothelial hyperplasia, possibly manifesting as clubbing of fingers and toes. VEGF receptors are expressed by bone-forming cells, promoting endochondral bone formation as well as stimulation and migration of osteoblasts. These features may explain the histologic alterations underlying HPO such as vascular hyperplasia, edema, and fibroblast/osteoblast proliferation [17].

Respiration 2012;83:357-362 


\section{Conclusions}

We here present the first case of PMML associated with HPO, the diagnosis being made on clinical, radiological, and pathological findings. Prominent physical findings included decreased respiratory sound and rales of the right lung, and digital clubbing of the extremities. Contrast-enhanced CT scan of the thorax demonstrated that the orifice of the right middle lobe bronchial lumen was subtotally obstructed by an irregular mass, with significant narrowing of the bronchial lumen. Histopathological examination showed sheets of spindle-to-pleomorphic cells with vesicular nuclei, prominent nucleoli, and brown cytoplasmic pigment. Immunohistochemistry showed malignant cells positive for $\mathrm{S} 100$ and $\mathrm{HMB} 45$, whereas cytokeratin, chromogranin, and CD68 were negative. With all the above features, we concluded that the diagnosis is PMML associated with HPO.

\section{Acknowledgments}

This report was supported by the National Natural Science Foundation of China (30971306/H0107), the 3rd program of National Project '973' of China.

\section{Disclosure Statement} terest.

The authors report no personal or financial conflict of in-

\section{References}

1 Kundranda MN, Clark CT, Chaudhry AA, Chan V, Daw HA: Primary malignant melanoma of the lung: a case report and review of the literature. Clin Lung Cancer 2006;7:279281.

-2 Maddipatla S, Pennock G, Magill MJ Jr, Nasser NM, Herrera LJ: A rare case of endobronchial amelanotic melanoma. J Thorac Oncol 2011;6:643-644.

>3 Thiberville L, Salaün M: Bronchoscopic advances: on the way to the cells. Respiration 2010;79:441-449.

4 Maeda R, Isowa N, Onuma H, Miura H, Tokuyasu H, Kawasaki Y: Primary malignant melanoma of the lung with rapid progression. Gen Thorac Cardiovasc Surg 2009; 57:671-674.

5 Testini M, Trabucco S, Di Venere B, Piscitelli D: Ileal intussusception due to intestinal metastases from primary malignant melanoma of the lung. Am Surg 2002;68:377-379.

6 Reddy VS, Mykytenko J, Giltman LI, Mansour KA: Primary malignant melanoma of the lung: review of literature and report of a case. Am Surg 2007;73:287-289.
7 Miller DL, Allen MS: Rare pulmonary neo plasms. Mayo Clin Proc 1993;68:492-498.

$\checkmark 8$ Rooney C, Sethi T: The epithelial cell and lung cancer: the link between chronic obstructive pulmonary disease and lung cancer. Respiration 2011;81:89-104.

-9 Jennings TA, Axiotis CA, Kress Y, Carter D: Primary malignant melanoma of the lower respiratory tract. Report of a case and literature review. Am J Clin Pathol 1990;94:649655.

$>10$ Pilozzi E, Cacchi C, Di Napoli A: Primary malignant tumour of the lung with neuroendocrine and melanoma differentiation. Virchows Arch 2011;459:239-243.

11 Armstrong DJ, McCausland EM, Wright GD: Hypertrophic pulmonary osteoarthropathy (HPOA) (Pierre Marie-Bamberger syndrome): two cases presenting as acute inflammatory arthritis. Description and review of the literature. Rheumatol Int 2007; 27:399-402.

12 Izumi M, Takayama K, Yabuuchi H, Abe K, Nakanishi Y: Incidence of hypertrophic pulmonary osteoarthropathy associated with primary lung cancer. Respirology 2010;15: 809-812.
13 Thompson MA, Warner NB, Hwu WJ: Hypertrophic osteoarthropathy associated with metastatic melanoma. Melanoma Res 2005; 15:559-561.

14 Ito T, Goto K, Yoh K, Niho S, Ohmatsu H, Kubota K, Nagai K, Miyazaki E, Kumamoto T, Nishiwaki Y: Hypertrophic pulmonary osteoarthropathy as a paraneoplastic manifestation of lung cancer. J Thorac Oncol 2010;5:976-980.

$>15$ Olán F, Portela M, Navarro C, Gaxiola M, Silveira LH, Ruiz V, Martínez-Lavín M: Circulating vascular endothelial growth factor concentration in a case of pulmonary hypertrophic osteoarthropathy. Correlation with disease activity. J Rheumatol 2004;31:614616.

16 Dickinson CJ: The aetiology of clubbing and hypertrophic osteoarthropathy. Eur J Clin Invest 1993;23:330-338.

17 Silveira LH, Martínez-Lavín M, Pineda C, Fonseca MC, Navarro C, Nava A: Vascular endothelial growth factor and hypertrophic osteoarthropathy. Clin Exp Rheumatol 2000;18:57-62. 